\title{
HALINA MANIKOWSKA
}

Instytut Historii im. Tadeusza Manteuffla Polskiej Akademii Nauk E-Mail: hmanik@supermedia.pl

\section{MAREK SŁON}

Instytut Historii im. Tadeusza Manteuffla Polskiej Akademii Nauk

E-Mail: marek.slon@wp.pl

\author{
ÜBER ZWEI DIMENSIONEN HINAUS \\ DIE SAKRALE GEOGRAFIE DER MITTELALTERLICHEN \\ STADT „NACH INNEN“ UND „NACH OBEN“
}

Der vorliegende Aufsatz geht auf eine Tagung und ihre Forschungsthemen zurück, entspricht aber nur in geringerer Maße dem damals gehaltenen Referat ${ }^{1}$. Die Organisatoren der Tagung haben ihr Thema auf eine Weise formuliert und dargelegt, die den Mediävisten nur scheinbar ein weites Feld für die Behandlung der Probleme bietet. Und faktisch stellt sie uns sogar an drei Stellen vor schwer zu bewältigende intellektuelle Herausforderungen. Diese Stellen wollen wir aufzeigen. Die erste betrifft schon die Formulierung des Tagungstitels: Zwischen Sacrum und Profanum. Sakrale Topografie der Stadt in Mitteleuropa. Wie ist die Kategorie „sakrale Topografie der Stadt“ zu verstehen, wie

1 Die Tagung unter dem Titel Zwischen Sacrum und Profanum. Sakrale Topografie der Stadt in Mitteleuropa fand in Würzburg vom 19. bis zum 20. September 2016 statt, s. http://historicus.umk.pl/pmh/strona/index.php?page=konferencja-zwischensacrum-und-profanum\&hl=de_DE (besucht am 23.02.2017). 
soll diese ,sakrale Topografie“ definiert werden, die sich irgendwo $d a$ zwischen befinden soll: als nicht völlig sakral und nicht völlig profan? Oder vielleicht zugleich (zumindest hinsichtlich der Funktion, aber nicht unbedingt der Zeiteinheit) sakral und profan? Dabei hat sich doch, unter anderem dank vielerlei Deutungen, das statische Bild der sakralen Punkte im Stadtplan in den letzten Jahrzehnten in ein vielschichtiges System städtischer religiöser Praktiken, städtischer Kulte und religiöser Feiertage verwandelt, aus denen sich ein dynamisches und fließendes Bild des städtischen sakralen Raumes ergibt. In diesem Bild kommt die dichotomische Konzeption von sacrum und profanum überhaupt nicht oder nur mit großer Mühe zur Anwendung. Aus diesem städtischen Raum werden dagegen geschlossene, mehr oder weniger abgegrenzte loci herausgegriffen, um sie total zu untersuchen, wobei auch ein methodologischer Eklektizismus nicht vermieden wird, wie im opus vitae von Dominique Iogna-Prat (La Maison Dieu. Une histoire monumentale de l'Église au Moyen Âge $)^{2}$.

Die Schwierigkeiten nehmen zu, wenn wir uns das Forschungsziel der erwähnten Tagung vergegenwärtigen, das nur auf den ersten Blick klar definiert zu scheint sein. Die Rede ist vom „Aufzeigen der Rolle der Bebauung mit religiöser Bedeutung für die Entstehung und Entwicklung der Städte in Mitteleuropa"3. Soll man etwa annehmen, dass die Bebauung mit religiöser Bedeutung (was immer das auch bedeuten mag) dasselbe ist wie die sakrale Topografie? Wenn das Gebäude, d.h. die materielle, physische Gestalt einer kirchlichen (religiösen) Institution einen Einfluss ausüben soll, muss es sich dann um den Einfluss seiner Architektur und des Materials, aus dem es errichtet wurde, auf die anderen Gebäude der Stadt handeln und über ihre urbanistische und architektonische Landschaft (denn doch wohl nicht über ihre Ge-

2 D. Iogna-Prat, La Maison Dieu. Une histoire monumentale de l'Église au Moyen Âge (v. 800-v. 1200), (2006).

3 http://historicus.umk.pl/pmh/strona/index.php?page=konferencja-zwischensacrum-und-profanum\&hl=pl_PL (besucht am 23.02.2017). 
stalt) entscheiden? Oder vielleicht nur über ihre Größe, auch im Sinne von Überheblichkeit? In Brüssel, wo die Kathedrale auf der Anhöhe des Teurenbergs errichtet wurde und ihre beiden Türme (64 Meter hoch) die im Tal gelegene Stadt überragen, muss der Rathausturm (aus dem 15. Jahrhundert) 96 Meter hoch sein, um diese Anmaßung zur übertreffen. In Breslau stellen, wie wir vermuten, die beiden Türme der Hauptpfarrkirche St. Maria Magdalena auch eine Antwort auf die beiden Türme der vor der Stadtmauer entfernt liegenden Kathedrale dar. Es genügt, die mittelalterlichen Stadtpläne zu betrachten - ob das nun rein symbolische sind (wie der berühmte Stadtplan von Jerusalem aus dem 12. Jahrhundert ${ }^{4}$ ) oder die bereits aus dem ausgehenden 15. Jahrhundert stammenden Panoramakarten - sie alle sind bemüht, an erster Stelle die Hierarchie der Bauwerke und ihre Lokalisierung wiederzugeben, eine im modernen Stadtplänen gar nicht mehr greifbare dritte, vertikale Dimension, die diese Hierarchie markiert. Sogar der außergewöhnliche Cambrai-Stadtplan von Jerusalem ${ }^{5}$, der mit dem Grundriss der Stadt operiert, auch wenn dieser idealisiert wird, bleibt seinem Zweck treu - hier wird die Hierarchie der Bauwerke vor allem durch ihre Größe veranschaulicht. Die bestens bekannten Stadtansichten aus der erstmals 1493 herausgegebenen Schedelschen Weltchronik geben in ihrem verblüffenden Schematismus an erster Stelle den städtischen Charakter der Stadt wieder, ihre Politeia, und verdrängen die Abbildung des Stadtpanoramas in den Hintergrund der Perzeption ${ }^{6}$. Erst die Städteprospekte, von denen manche älter sind als diese Holzschnitte, wie die Panoramakarte von Florenz mit Anwen-

4 Die sogenannte illustrierte Bibel von Den Haag, Koninklijke Bibliotheek den Haag, KB, 76 F 5, fol. 1r: http://manuscripts.kb.nl/show/images_text/76+F+5/page/1 (besucht am 23.02.2017).

5 S. http://www.qantara-med.org/qantara4/public/show_document.php?do_ id=1267 (besucht am 14.3.2017).

${ }^{6}$ K. Arnold, Bilder und Texte. Stadtbeschreibung und Städtelob bei Hartmann Schedel, in: Acta Conventus Neo-Latini Hafniensis. Proceedings of the Eighth International Congress of Neo-Latin Studies, (Medieval Texts and Studies 120, 1994), S. 121-132. 
dung der Perspektive und die Vogelperspektive der berühmten Carta (oder Pianta) della catena (die auf die Jahre 1472-1481 datiert und Francesco di Lorenzo Rosselli zugeschrieben wird), sind für uns mühelos lesbar. Aber das sind Zeugnisse des Umbruchs, der sich im 14.-15. Jahrhundert im Begreifen und Wahrnehmen des Raumes überhaupt vollzog ${ }^{7}$. Die damals wiederentdeckte lineare Perspektive ging mit der Überzeugung einher (die bald von Leon Battista Alberti wissenschaftlich bewiesen wurde), dass es möglich ist, die Dreidimensionalität in zwei Dimensionen abzubilden, und dass gerade die lineare Perspektive die materiellen Räume authentisch wiedergibt. Was nicht bedeutet, dass die Autoren der Stadtpläne bei der Anwendung der Perspektive die physische Wirklichkeit nicht manchmal „verfälschten“, um zum Beispiel die politischen oder ideologischen Realitäten hervorzuheben so vergrößerten sie manche Gebäude, versetzten sie auf dem Stadtplan an eine andere Stelle und dergleichen aus, um ihre Bedeutung (oder genauer gesagt: die ihres Inhalts) wiederzugeben.

In Anerkennung dessen, dass ein effektiver Umgang mit derartigen Fallstricken auf ihrer Wahrnehmung - oder am besten auf ihrer Entschärfung - beruht, beschlossen wir, uns in diesem Artikel mit der Problematik der "sakralen Topografie“ zu beschäftigen, verstanden als eine Kategorie sozialer Praxis, eine Kategorie der Vorstellung und der Perzeption, die in zumindest wesentlichem Grade Einfluss auf die "Gestalt der Stadt" hat. Wir werden uns auf verschiedene, in den Forschungen der Historiker präsente Theorien berufen, an erster Stelle auf die Theorie des sozialen Raumes von Henri Lefebvre und die in der Strömung des sogenannten spatial turn präsenten Theorien ${ }^{8}$.

Begonnen werden muss jedoch mit den terminologischen Fragen als Kernproblem und der banalen, aber keineswegs marginalen Bemer-

7 L. Bek, The Changing Architectonic Aspect of the Ideal City in the Early Renaissance, in: ebenda, S. 143-153; D. Friedman, 'Fiorenza' Geography and Representation in a Fifteenth Century City View, „Zeitschrift für Kunstgeschichte“, 64 (2001), S. 56-77.

8 H. Lefebvre, La production de lespace, (1974). 
kung, dass der sakrale Raum (die sakrale Topografie) auf zweifache Weise entsteht: durch Sakralisierung eines bestimmten Raumes und/ oder durch ein bestimmtes Arrangieren der Heiligkeit (des Heiligtums) im Raum. Die sich mit sakraler Topografie beschäftigenden Historiker, besonders diejenigen, die diese auf eine statisch und enumerativ verstandene Verteilung der kirchlichen Gebäude (Institutionen) in der Stadt reduzieren, ziehen nur selten die damalige Konzeptualisierung des Raumes und die Art und Weise der Bestimmung eines Platzes in ihm in Betracht. „Locus est ubi sit, in foro, in platea“, stellte Isidor von Sevilla verbindlich für die Menschen der nächsten Jahrhunderte fest ${ }^{9}$, und die Lage kann sechs Richtungen entsprechend beschrieben werden, welche bei dieser Gelegenheit bewertet werden und eine Rangordnung bekommen: nach rechts, nach links, nach vorn, nach hinten, nach oben, nach unten, sowie in Bezug auf den als Bezugspunkt fungierenden Ort: nahe gelegen (prope), weiter weg (longe) oder um etwas herum (terra circa monasterium posita). Fast völlig übergangen wird auch die Vieldeutigkeit (in allen Sprachen) des Adjektivs sacrale.

Isidor definierte den Unterschied zwischen drei Kategorien (Termini), die für diese Tagung besonders wichtig sind: „Sacrum uocamus quod ad Deum pertinet, religiosum quod ad homines iustos, sanctum uero quod ad aliquid sancitur quoque uiolato poena comittitur. Sacrum uero et sanctum est, sanctum uero non continuo sacrum "10. Das, was sacrum ist, ist also auch sanctum, aber umgekehrt gilt dies nicht ${ }^{11}$. Das Wort religiosus besaß schon damals, zur Zeit Isidors, die Bedeutung

9 Isidori Hispalensis episcopi Etymologiarum sive Originvm libri XX, hg. v. W. M. Lindsay, 1 (1911), Lib. II, cap. XXV De categoriis Aristotelis, 8.

10 C. Codoñer, Isidoro de Sevilla. Diferencias, libro I. Introducción, edición crítica, traducción y notas, (Auteurs Latins du Moyen Âge 1, 1992), I, 33.

11 Wegen der Ambivalenz des Wortes sacrum, das „duo significat, et bonum et malum; bonum ut illud: «Inter flumina nota et fontes sacros»; malum, ut: «Auri sacra fames» et «Sacre panduntu portae», «et leno sacer et sacer hircus» “ usw., während sich das sanctum immer als das Gute platziert. 
,Bischöfe und Mönche, die ihm der hl. Augustinus verliehen hatte ${ }^{12}$, der mit diesem Wort Personen bezeichnete - Christen, Gerechte (jene iusti) mit beispielhafter Lebensführung. Isidor „, christianisierte“ Begriffe, die eine heidnische Herkunft besaßen ${ }^{13}$, indem er - generell genommen distinktiv Objekte definierte, die dem göttlichen Gesetz unterstanden und niemandem gehörten (niemandes Eigentum bildeten), aber einen (auch rechtlich) ungleichen Status besaßen: der Tempel ist feierlich Gott geweiht und heiligt den Ort, auf dem er steht, selbst wenn er zerstört wird; heilige (sancti) Orte - Mauern und Tore - sind nicht in demselben Grade „heilig“ wie der Tempel oder der Altar; und zu noch einer anderen Kategorie (religiosus) gehört der Friedhof. Die Begriffe oder Kategorien sacer und religiosus besitzen ihre Gegensätze: profanus und purus, aber für sanctus gibt es keinen ${ }^{14}$.

Das römische Recht war selbstverständlich nicht die einzige Quelle für die Herausbildung einer christlichen, mittelalterlichen Konzeptualisierung der Sakralität des Raumes, der Orte und der Objekte. Sie vollzog sich, sehr vereinfacht gesagt, auf zweierlei Weise: die erste resultierte aus der Geburt des mit den Gräbern der Märtyrer verbundenen Konzepts locus sacer, die andere aus der gregorianischen Unterscheidung von spiritualia und temporalia. Die heiligen Orte und Gegenstände wurden zur Kategorie der spiritualia gezählt, mit allen sich daraus ergebenden Konsequenzen (konsekrierte Kirche und Altar, Reliquien).

Bis ins 3. Jahrhundert hinein wehrte sich das Christentum dagegen, physischen Orten den Charakter der Heiligkeit zu verleihen. Eine Veränderung brachte erst die Verehrung der Märtyrergräber (damals erschien in den Quellen der Begriff locus sacer) und die Bautätigkeit

12 Ganz anders als das römische, heidnische, rechtliche, das u.a. auch Leichen und Friedhöfe umfasste.

13 M. Lauwers, La mémoire des ancêtres. Le souci des morts. Morts, rites et société au Moyen Âge, (1966), S. 220; M. de Souza, La question de la tripartition des catégories $d u$ droit divin dans l'Antiquité romaine, (2004). Die Dreiteilung im Codex Iustinianus war stark inspiriert von den Gaianischen Institutionen.

14 De Souza, ebd., S. 50. 
Konstantins, besonders in Jerusalem (erst damals eine heilige Stadt) und Bethlehem. Heilige Orte waren also Gott und seinen Dienern geweihte, von heiligen Männern oder Mönchen bewohnte Orte und deren Grabstätten. Im Frühmittelalter wurde das Kloster durch Antonomasie zum heiligen Ort - die Wahl des Ortes für das Kloster arrangiert Gott, die Stiftung „reinigt“ die Umgebung und redefiniert selbstverständlich auch die Beziehungen zwischen den Menschen und dem Raum.

Als das römische Recht ab dem 12. Jahrhundert sogar außerhalb des ehemaligen römischen Limes immer größeren Einfluss auf die Rechtssysteme bekam, gewann die oben erwähnte römische Definierung heiliger Orte und Objekte immer mehr an Bedeutung. Jean Beleth teilte in seiner Summa de ecclesiasticis officiis die Gebetsorte ein in sacra, sancta und religiosa ${ }^{15}$. Hier ist nicht der Platz, sich näher mit dieser Dreiteilung zu befassen; es soll lediglich darauf aufmerksam gemacht werden, dass durch ihre Vernachlässigung in den Forschungen der Begriff der sakralen Topografie verflacht und die richtige Erfassung aller ihrer Elemente erschwert wird, darunter der wichtigsten: der hierarchischen und der symbolischen.

Bei der Erforschung der sakralen Topografie der Stadt ist die Verflechtung der Wahrnehmungen, Vorstellungen und Ideen mit dem physischen, messbaren Raum und mit materiellen Objekten zu beachten. In der Forschung der letzten Jahre wurde zurecht betont, dass der Raum nicht direkt auf das menschliche Handeln wirkt, sondern nur durch seine Wahrnehmung. Sie ist von vielen Faktoren geprägt, subjektiv, von der früheren Erfahrungen gestaltet. Viel zu oft wird jedoch dabei außer Acht gelassen, dass der Raum selbst doch auf die Vorstellungen und Taten einen großen Einfluss hat ${ }^{16}$. Seine Kraft, auch wenn von Menschen

15 Iohannis Beleth Summa de ecclesiasticis officiis, hg. v. H. Douteil, (Corpus Christianorum Continuatio Maedievalis 41 A, 1975), S. 5: „Locorum autem, que orationi sunt dicata, alia sunt sacra, alia sancta, alia religiosa" (ähnlich bei Wilhelm Durand).

16 Z. B. C. Hochmuth, S. Rau, Stadt - Macht - Räume. Eine Einführung, in: Machträume der frühneuzeitlichen Stadt, hg. v. C. Hochmuth, S. Rau, (2006), S. 13-40, hier 27-29; diesbezügliche Diskussion fasst Michael Pauly zusammen: M. Pauly, M. Scheutz, 
nicht wahrgenommen oder nicht verbalisiert, gibt ihnen feste Rahmen und steckt tief in jeder Idee. Dieses Gewicht des Raumes war in der vorindustriellen Zeit noch viel stärker als heute.

Diese Bemerkung betrifft auch die erwünschte Präzision beim Operieren mit der Chronologie und die Berücksichtigung des physischen Zustandes der diese Topografie konstruierenden Objekte. Bei der Wahl zwischen der synchronen und diachronen Erforschung und Darstellung einer Stadt soll der Schwerpunkt auf die erste gelegt werden. Wie wurde die Sakralität einer im Gewittersturm eingestürzten oder von einem Aggressor zerstörten Kirche wahrgenommen, die im Zustand einer Ruine oder ihres Wiederaufbaus manchmal mehrere Jahrzehnte auf die Weihe des Hauptalters warten musste? ${ }^{17}$ Kirchengebäude in mehr oder weniger ruinösem Zustand, im Umbau oder im Abriss begriffen, mit Löchern im Dach und einer teilweise eingestürzten Fassade oder Seitenwand, boten, wie alles darauf hindeutet, in mittelalterlichen Städten keinen seltenen Anblick, aber wir wissen nicht viel darüber, wie diese Gebäude im Alltag als Kirche funktionierten und wie ihre Sakralität wahrgenommen wurde. Die Krakauer St. Martins-Kirche brannte 1451 nieder und wurde erst 1541 wieder aufgebaut. Bildete sie fast hundert Jahre lang einen Bestandteil der sakralen Topografie Krakaus oder nicht?

Eine Antwort darauf fällt überhaupt nicht leicht. Die „sakrale Topografie" sollte als ein Prozess mit seiner eigenen Dynamik gesehen werden. Im Heiligen Land, in dem nach dem Untergang der Kreuzfahrerherrschaft sofort der Prozess des Verschwindens oder zumindest des Verfalls der materiellen Markierung (hauptsächlich durch Kirchenbauten) der Orte begann, die von Jesus, seiner Mutter und den Aposteln zeugten, erwies sich eine „legendäre Topografie“ als ausreichend, wie

Der Raum und die Geschichte am Beispiel der Stadtgeschichtsforschung, in: Cities and their Spaces: Concepts and their Use in Europe, hg. v. M. Pauly, M. Scheutz, (Stadtforschung A 88, 2014), S. 1-14.

17 Zur Rolle des Altars für die Sakralisierung des Ortes siehe insbesondere IognaPrat, La Maison Dieu. 
diese von Maurice Halbwachs treffend bezeichnet wurde ${ }^{18}$, eben um nicht nur die in den loca konkretisierte heilige Topografie in allen Einzelheiten zu bewahren, sondern um diese dann auch bedeutsam auszubauen. Der 14 Stationen zählende Kreuzweg auf einer genau abgesteckten, unveränderten Strecke, der heiligste Abschnitt des circuitus der Pilger, war das Ergebnis eben einer solchen apokryphen Ausschmückung einiger weniger Sätze aus dem Evangelium. Der fast 100 Jahre im Bau befindliche Petersdom in Rom scheint (zumindest aufgrund von Pilgerberichten) auch nicht für einen Augenblick irgendetwas von seiner ganz hoch auf der Skala angesiedelten Heiligkeit verloren zu haben.

Der Rückgriff auf römische oder Jerusalemer Beispiele als Analogien kann jedoch auch unbegründet sein, denn die Heiligkeit jener Orte und Kirchen, die Heiligkeit dieser Städte gründete sich gar nicht so sehr auf die materiellen Objekte als vielmehr auf die Geschichte und die Erinnerung. Im Christentum waren, wie Peter Brown stark hervorhob $^{19}$, bereits der Begriff heiliger Orte und deren Konzeptualisierung in beträchtlichem Maße das Ergebnis einer gewaltigen Bedeutung der Geschichte überhaupt in dieser Religion (als Erbe des Judaismus). Die Sakralisierung des Raumes war - an bestimmten Punkten - eine Materialisierung geheiligter Zeit (der heiligen Geschichte, des Heroismus der Märtyrer). Die Sakralisierung des Raumes war also seine Memorierung (Erinnerung).

Über die Stellung einer Kirche innerhalb der Hierarchie der Stadtkirchen entschied ihr institutioneller und liturgischer Rang. Aber es kam vor, dass sich in einigen Perioden des Kirchenjahres oder auch zyklisch alle paar Jahre ein außergewöhnlich mit Heiligtümern (Reliquien) gesättigter Ort ganz oben in der Hierarchie platzierte, zum

18 M. Halbwachs, La topographie légendaire des Évangiles en Terre sainte. Étude de mémoire collective, (1941).

19 P. Brown, The Cult of the Saints: Its Rise and Function in Latin Christianity, (1981). 
Beispiel ein Pilgerort, der dann im Spätmittelalter die Gläubigen in der Regel auch durch attraktive Ablässe anzog. Dabei brauchte es sich gar nicht um ein ansehnliches Kirchengebäude oder Klosterensemble zu handeln. Die großen ostensiones reliquiarum, die im Spätmittelalter in unserer Region in einigen Städten organisiert wurden (um nur die wichtigsten zu nennen: in Prag, Nürnberg, Halle, Hall, Wien, Bamberg, Wittenberg, Würzburg, Regensburg, Breslau), fanden sowohl in der Kirche als auch vor ihr sowie auf speziellen, eigens zu diesen Anlässen errichteten Türmen statt.

Die Phänomenologie des Sanktuariums ist ziemlich komplex; sie veranschaulicht, wie sich das Sacrum allmählich den Raum um den Altar herum und auch außerhalb von ihm aneignete ${ }^{20}$. Der Übergang vom Konzept des heiligen Ortes zum Konzept des sakralen Raumes vollzieht sich zwischen dem 11. und dem 13. Jahrhundert, das heißt parallel zu solchen sozialen Prozessen und kulturell-religiösen Veränderungen wie den neuen Formen des Siedlungswesens und der Erfassung der Bevölkerung (Pfarrei, Dorf und Stadt in ihrer neuen topographischen und urbanistischen Gestalt), des Auftretens und der schnellen Verbreitung einer ausgebauten Topografie des Jenseits (Paradies, Purgatorium, Hölle, Limbus der Patriarchen, Limbus der Kinder, Abrahams Schoß) und der Rückkehr der euklidischen Geometrie (lateinische Übersetzung des Abelard von Bath).

Systematische, problembezogene, von methodologischer Reflexion begleitete Untersuchungen von Historikern über die räumliche Dimension des religiösen Kults begannen erst richtig mit den Studien zur Hagiographie. Es genügt zu erwähnen, dass der technische Begriff loca sanctorum von Hyppolite Delehaye ${ }^{21}$ in die wissenschaftliche

20 Vgl. u.a.: Luoghi sacri e spazi della santità, hg. v. S. Boesch Gajano, L. Scaraffia, (1990); D. Méhu, Locus, transitus, peregrinatio. Remarques sur la spatialité des rapports sociaux dans l'Occident médiéval (Xie-XIIIe siècle), in: Construction de l'espace au Moyen Âge: pratiques et représentations. XXXVIIe Congrès de la SHMES (Mulhouse, 2-4 juin 2006), (2007), S. 275-293.

21 H. Delehaye, Loca sanctorum, (1930). 
Historiographie eingeführt wurde, und dass die Heiligenverehrung im Zusammenhang mit der Sozial- und der Kulturgeschichte sowie die Dimension der Heiligkeit seit den sechziger Jahren von Gelehrten aus dem Kreis um Gabriel Le Bras und Évelyne Patlagean untersucht wurden. Daher verwundert es nicht, dass in der polnischen Mediävistik die Problematik einer nicht auf die Topografie der Kirchenbauten reduzierten sakralen Topografie von einer Erforscherin von Heiligenviten, von Aleksandra Witkowska, aufgegriffen wurde ${ }^{22}$, die von französischen Forschungen und dem Konzept der sakralen Geografie inspiriert war. Dieses Konzept hatte schon in der Mitte des 19. Jahrhunderts seine entsprechende Rechtskraft und feierliche Bestätigung gewonnen, als man mit der Herausgabe des monumentalen enzyklopädischen Werkes Dictionnaire de géographie sacrée et ecclésiastique begann. Im vergangenen Jahrhundert evoluierte dieses Konzept dann unter dem Einfluss der Religionskunde, der Religions- und Kulturanthropologie sowie der Kulturgeografie mit ihrem Nachdruck auf den Raum der Kommunikation und die symbolischen Aktivitäten, und schließlich auch noch unter dem Einfluss der Theorien von Forschern, für die die Kategorie des Raumes im Zentrum ihrer Abhandlungen stand: von Heidegger, Cassirer, Foucault oder de Certeau, um nur die wichtigsten zu nennen.

Die ausdrückliche semantische Unterscheidung des Ortes (lieu) vom Raum (espace) durch de Certeau ist wichtig für den eine mittelalterliche Stadt erforschenden Historiker ${ }^{23}$. Ein Ort ist derjenige Teil der Umgebung (endroit), in welchem die Dinge und Objekte nach einer bestimmten Ordnung (statisch, stabil) untergebracht sind, während ein Raum ein benutzter, in Bewegung befindlicher Ort ist, der seine Richtung und zeitliche Beschränkung besitzt. Dies begründet den we-

22 A. Witkowska, Kulty patnicze piętnastowiecznego Krakowa. Z badań nad miejską kulturą religijną, (1984).

23 M. de Certeau, L'Invention du quotidien, 1: Arts de faire (1990); siehe insbesondere Kapitel VII Marches dans la ville sowie Kapitel IX Récits d'espace. 
sentlichen Unterschied zwischen der geographischen Landkarte und Topografie (die die Orte stabil wiedergibt) und der „wirklichen“ (in Bewegung befindlichen) Geografie und dem (fließenden) Raum. In den neueren Forschungen mit historischem Profil unterscheidet sich der Begriff der "sakralen Geografie“ eben dadurch vom Begriff der „sakralen Topografie " und insbesondere der „kirchlichen (institutionell-sozialen) Topografie", dass er die Perzeption (die Philosophie der Perzeption) von Raum und Zeit betont, eine Analyse der Beziehungen zwischen diesen Kategorien voraussetzt und sich auf symbolische Hierophanien konzentriert. In Bezug auf die Forschungen zur religiösen Kultur des europäischen Mittelalters bedeutete dies für lange Zeit eine gleichsam natürliche Privilegierung der Forschungen über Pilgerorte. Die Ausweitung dieser Problematik ist dann das Verdienst der historischen Urbanistik und der historischen Anthropologie sowie der Studien zur politischen Kultur. In demselben Buch von Aleksandra Witkowska gehört das 2. Kapitel „Die loca sacra Krakaus im 15. Jahrhundert" mit den der kirchlichen Topografie und dem Bau neuer Pilgerorte (an den Gräbern von Ordensleuten, des Bischofs und der Königin, die als Heilige galten, aber ohne kanonisierende Sanktionierung) gewidmeten Abschnitten in konkreten Kirchen zur sakralen Topografie; das darauffolgende, dem Funktionieren dieser Zentren gewidmete Kapitel gehört zur sakralen Geografie, wo der concursus populi wichtig ist, und wahrgenommen wird der Einfluss der in die Städte einströmenden Menschenmassen (darunter solcher mit Bewegungsbehinderung) auf ihre Landschaft und ihren Raum. Diese Erfahrung des Raumes ist auch den heutigen Bewohnern von Częstochowa nicht fremd, die sich bestens darüber im Klaren sind, dass nicht nur das die Stadt auf dem Hellen Berg (Jasna Góra) überragende Bauwerk der Klosterkirche Einfluss auf die Gestalt ihrer Stadt hat, sondern auch (vielleicht sogar hauptsächlich) der im Laufe des Jahres fast stetige Zustrom von Hunderttausenden von Pilgern.

Das bedeutet selbstverständlich, dass zwar die Kathedrale, die Pfarrkirchen, Klosterkirchen, Oratorien, Altäre und Prozessionswege 
ein Netz der wichtigsten materiellen Elemente sakraler „Bebauung“ schaffen, die sakrale „Besiedlung“ der Stadt aber genauso wesentlich ist. Eine besondere Rolle spielen hierbei manche Gebetsgemeinschaften (Chorherren und Nonnen) sowie der concursus populi, der das Funktionieren der loca sacra konstituiert. Jede Veränderung dieser „Bebauung“ und dieser „Besiedlung “ bedeutet eine Veränderung der sakralen Geografie sowie eine Veränderung der Raumqualität. Die sakrale Geografie kann unter bestimmten Umständen außerhalb oder über der Topografie der Stadt existieren, so als ob sie aus ihr herauspräpariert worden wäre, wovon die Berichte von Pilgern nach dem Heiligen Land über ihren Aufenthalt in Venedig oder die spätmittelalterlichen Pilgerführer über Rom zeugen; in beiden Fällen betreffen die Schilderungen aus dem städtischen Gewebe, aus seiner Landschaft „herauspräparierte“ Sanktuarien und Reliquien.

Seit den siebziger Jahren des 20. Jahrhunderts werde zur Analyse der Stadtplanung und Stadttopografie die Kategorie des konzipierten Raums benutzt - im Sinne des Lefebvre'schen espace conçu: eines Trägers von Bedeutungen, die ihm mit Hilfe der Lokalisierung, Bebauung, plastischen und verbalen Umrahmung, der Funktion und des Funktionierens der einzelnen Objekte verliehen wurden. Damit würden sich diese Forschungen denen der Kunsthistoriker annähern, die ihre, immer in einem Raum existierenden, Forschungsobjekte schon beträchtlich früher ,ideologisiert“ haben. Lange privilegiert blieb hier die sakrale Topografie ${ }^{24}$. Auf der Grundlage neuer Studien, die nach tieferen Interpretationen suchten und sich nicht immer des Jargons

24 Siehe insbesondere die diese Forschungen in Polen initiierenden Arbeiten von M. Morelowski, Początki świadomej myśli urbanistycznej w Polsce przed kolonizacją XIII w., in: Sztuka i historia. Księga pamiattkowa ku czci profesora Michała Walickiego, (1966), S. 39; R. Michałowski, Princeps fundator. Studium z dziejów kultury politycznej w Polsce X-XIII wieku, (1993); K. Skwierczyński, Custodia civitatis. Sakralny system ochrony miasta $w$ Polsce wczesnego średniowiecza na przykładzie siedzib biskupich, „Kwartalnik Historyczny“, CIII, 3 (1996), S. 3-51; A. Pleszczyński, Przestrzeń i polityka. Studium rezydencji władcy wcześniejszego średniowiecza. Przykład czeskiego Wyszehradu, (2000). 
des spatial turn bedienten, gelang es, die Hauptelemente der mittelalterlichen urbanistischen Konzeptionen zu unterscheiden, zu denen vor allem Nachahmung und Angleichung (an Rom, an Jerusalem) gehören ${ }^{25}$.

Ein im Früh- und Hochmittelalter in Europa verbreitetes Phänomen bestand darin, dem vom Menschen organisierten, von Kirchen und den sich in ihnen befindlichen Reliquien geheiligten Raum symbolische Bedeutungen und sakrale Kraft zu verleihen. Der besonderen Bedeutung und Funktion einer so verstandenen sakralen Topografie waren sich die Stifter ganz gewiss bewusst: die Bischöfe und Herrscher sowie - vielleicht sogar vor allem - die über sie und ihre Werke berichtenden Chronisten und Hagiographen ${ }^{26}$. Die von ihnen geschilderten Bischofs- und Klostersitze erscheinen als vollständig durchdachte und konsequente verwirklichte Gründungen. Dank der Stiftungen Heinrichs II., des Bischofs Otto, seiner Nachfolger und des Ritters Reginold war Bamberg beispielsweise ,sic locus Babenbergensis aecclesiis et patrociniis sanctorum in modum crucis undique munitus ${ }^{\text {"27. Dabei muss }}$ angemerkt werden, dass sich die urbanistisch-topographische Ausführung hier nicht auf der Größe oder der Architektur der Kirchenbauten gründet, sondern auf ihrer Lokalisierung zueinander sowie auf ihren patrocinia. Eine solche Konzeptualisierung und Perzeption sakraler Topografie eröffnete ein weites Feld für den Griff nach Repliken und die

25 Literatur zu diesem Thema in: H. Manikowska, Sacred Geography of a Town, „Acta Poloniae Historica“, 101 (2010), S. 51-87.

26 Eine Anthologie derartiger Texte bieten die Schriftquellen zur Kunstgeschichte des 11. und 12. Jahrhunderts für Deutschland, Lotharingen und Italien, erarb. v. O. Lehmann-Brockhaus, (1938); vgl. auch: J. von Schlosser, Quellenbuch zur Kunstgeschichte des abendländischen Mittelalters, (1896); V. Morlet, Recueil de textes relatifs à l'histoire de l'architecture et à la condition des architectes en France au moyen âge, XI-XII siècles, (1911).

27 Adalberti Vita Heinrici II imperatoris, hg. v. G. Waitz, in: Annales, chronica et historiae aevi Carolini et Saxonici, hg. v. G. H. Pertz, (Monumenta Germaniae Historica, Scriptores 4, 1841), S. 794, cap. 7. 
Nachahmung der berühmtesten (und gleichzeitig heiligsten) Objekte (wie der Grabeskirche in Jerusalem) und Städte.

Die Errichtung kirchlicher Gründungen ad instar, ad similitudinem in den Zentren mit höchster (tatsächlicher oder erst postulierter) politischer und ideologischer Bedeutung war besonders dort von Nutzen, wo es entweder an „eigenen“ lokalen Heiligen mangelte oder diesen kein ausreichend hoher Rang verliehen werden konnte, aber dies geschah auch in frisch christianisierten Ländern. Besonders deutlich trifft dies auf Polen zu, einer wahren Wüste vor Ende des 12. Jahrhunderts und sogar noch in der Mitte des darauffolgenden Jahrhunderts, was Sanktuarien mit Gräbern eigener Heiliger betrifft. Der Erfolg, den die Errichtung eines auf der Anhäufung von Heiligtümern basierenden Sanktuariums an der Wende vom 10. zum 11. Jahrhundert in Gnesen darstellte, erwies sich als kurzlebig. Um 1039 raubte der böhmische Fürst Břetislav, nachdem er Gnesen zerstört hatte, die wichtigsten Heiligtümer und brachte sie nach Prag: die Reliquien des hl. Adalbert und die der Fünf Heiligen Brüder. In Polen mangelte es sogar an einer „anständigen “ Zahl spektakulärer Reliquientranslationen ${ }^{28}$. Die an der Wende vom 11.zum 12. Jahrhundert wichtigste Kathedrale - in Krakau - besaß in ihrer Schatzkammer sechs oder vielleicht (höchstens) acht Reliquiare. In Böhmen sind Berufungen auf Rom sehr deutlich erkennbar. Cosmas, der wichtigste böhmische Chronist des Hochmittelalters, nahm an, dass der hl. Wenzel, der Patron des Königreiches, den Bau der St. Veits-Rotunde nach dem Vorbild einer römischen Kirche initiierte. Auf römische Vorbilder soll sich auch König Vratislav II. (1061-1092) berufen haben, als er seinen Sitz auf den nahegelegenen WyschehradHügel verlegte, nachdem sein Bruder Jaromír (mit Ansprüchen auf Teilhabe an der weltlichen Herrschaft) Bischof von Prag und damit ein

$28 \mathrm{Zu}$ den wichtigsten und - was wesentlich ist - auch gesicherten gehören: die Translation der Reliquien des hl. Vinzenz, des Bischofs von Chieti, aus Magdeburg in das Benediktinerkloster in Breslau, einer Stiftung des mächtigen Magnaten Piotr Włostowic, im Jahre 1145 sowie die Translation der Reliquien des hl. Florian nach Krakau im Jahre 1184. 
allzu naher Nachbar des Fürsten auf der Burg geworden war. Vratislav stiftete auf dem Wyschehrad ein Domkapitel, für das er eine Exemtion vom Ortsbischof und die direkte Abhängigkeit vom Papst erwirkte. Die Imitation betraf nicht die Architektur der römischen Basilika, sondern den kaiserlichen Akt der Stiftung des christlichen Roms: „Et in die fundationis Wratislaus rex 12 cophinos lapidum ad modum quondam Constantini imperatoris, propriis humeris suis portans, aedificorum prima posuit fundamenta, et Wyssogradensem ecclesiam ad similitudinem ecclesiam Romanae s. Petri construxit [...] in qua propriam sepulturam elegit" ${ }^{29}$.

Die untersuchten Beispiele aus dem 11. und 12. Jahrhundert (Krakau, Gnesen, Wyschehrad in Prag) zeigen, dass zur Gestaltung der sakralen Topografie dieser politischen, kirchlichen und ideologischen Zentren auf recht unterschiedliche Vorbilder wie Aachen (Krakau) oder Rom (Prager Wyschehrad) zurückgegriffen wurde und dass gleichzeitig die im Raum „untergebrachten“ Heiligen (ihre Patrozinien und Reliquien) diese imitatorische und aussagekräftige sakrale Topografie des Zentrums schufen und seinen Bewohnern ihre Protektion gewährten. In dieser Frühzeit der Städtegeschichte in den östlichen Randgebieten der abendländischen Kirche begannen die politisch-kirchlichen Zentren sicher auch auf andere Burgzentren als Vorbilder einzuwirken, indem

29 S. auch: Canonici Wissegradensis continuatio Cosmae, hg. v. J. Elmer, (Fontes rerum Bohemicarum 2, 1874), S. 201-237, hier S. 206-207: „[...] quod tali modo fundavit, portans propriis humeris XII cophinos plenos lapidibus in honore salvatoris, ut Constantinus imperator fundavit Romanam ecclesiam“. Vgl. Das Constitutum Constantini, hg. v. H. Fuhrmann, (Monumenta Germaniae Historica, Fontes iuris Germanici antiqui, in usum scholarum 10, 1968), S. 56-98, cap. 13: „construxisse nos [Konstantin] intro palatinium nostrum Lateranense eidem salvatori nostro domino deo Iesu Christo ecclesiam a fundamentis cum baptisterio, et duodecim nos sciatis de eius fundamentis secundum numerum duodecim apostolorum cophinos terra onustatos propriis asportasse humeris“. Diese Stiftung bildete unlängst den Gegenstand der Studien von Pleszczyński, Przestrzeń i polityka; V. Vaníček, Vratislav II. (I.) - První český král. Čechy $v$ době evropského kulturního obratu v 11. Století, (2004), S. 73-5, 106-111. 
sie ihnen „Patrozinienlisten“ und zumindest in gewissem Grade auch Modelle für die räumliche Anordnung der Kirchen lieferten.

Im Spätmittelalter wecken die „Transplantationen“ Jerusalems unsere Aufmerksamkeit. Am Beispiel dreier im Königreich Böhmen gelegener Städte - Prager Neustadt, Breslau und Görlitz - kann aufgezeigt werden, dass es sich hierbei um ein Phänomen handelte, das im 14.-15. Jahrhundert noch verschiedene Formen annahm, auch unterschiedliche Inhalte enthielt und nur in einem der analysierten Fälle (Görlitz) anerkannterweise zur Entstehung einer topografischreligiösen Gründung führte, die wir unter dem Namen Neues Jerusalem, Kalvarienberge, Sacri Monti kennen. In ihnen finden wir drei hinsichtlich der Form, der Entstehungszeit, der Struktur und der Funktion verschiedene „Verlagerungen“ Jerusalems, die den verschiedenen Etappen und Weisen der Perzeption des historischen Jerusalems im Mittelalter entsprechen.

Die Prager Neustadt wurde von Karl IV. in den Jahren 1347-1348 als vierte besondere Stadtgemeinde innerhalb der Prager Agglomeration (nach der Prager Altstadt, der Kleinseite [Malá Strana] und dem Hradschin [Hradčany]) bald nach zwei über die Zukunft Karls IV. entscheidenden Ereignissen gegründet: nach seiner Wahl zum römischdeutschen König im Juli 1346, d.h. de facto zum Kaiser, sowie nach dem Tod seines Vaters Johann von Luxemburg in der Schlacht von Crécy am 25. August desselben Jahres ${ }^{30}$. Das Zentrum von Karls Herrschaft Prag - musste in den Rang der wichtigsten Stadt des Reiches erhoben werden und diesen Rang auch manifestieren können. Diesem Zweck

30 Vgl. V. Kotrba, Nové Město pražské: „Karlstadt“ $v$ univerzální koncepci císaře Karla IV., in: $Z$ tradic slovanské kultury v Čechách (Sázava a Emauzyv dějinách české kultury), hg. v. J. Petr, S. Šabouk, (1975), S. 53-66. Die umfangreichste Studie über die Stiftung der Neustadt und ihre Raumgestaltung bietet V. Lorenc, Nové Město pražské, (1973) und ders., Das Prag Karls IV: Die Prager Neustadt, (1982): auf seine Befunde gründen sich meine Ausführungen zum großen Teil. Vgl. auch zuletzt V. Ledvinka, J. Pešek, Prag, (2000), besonders S. 144; sowie Nové Město pražské ve 14.-20. století, hg. v. V. Ledvinka, J. Pešek, „Documenta Pragensia“, 17 (1998). Die gesamte Prager Agglomeration nahm etwa 747 ha ein (106 ha die Altstadt). 
diente auch die von Karl schon vorher in Avignon erkämpfte Erhebung des Prager Bistums in den Rang eines Erzbistums (1344) sowie die Stiftung einer Universität in Prag im Jahre $1348^{31}$.

Die Prager Neustadt wurde nicht in der Ödnis gegründet. Unter anderem befanden sich dort schon ältere Kirchenbauten, deren Verteilung im Gelände - vom Gesichtspunkt der diesen Teil Europas spätestens seit dem 11. Jahrhundert erreichenden urbanistischen Wellen des Frühmittelalters - keineswegs als zufällig angesehen werden darf. Von entscheidender Bedeutung für die Manifestierung dieses in der Topografie der Neustadt und - in der Konsequenz - der gesamten Prager Agglomeration enthaltenen ideologischen Programms waren die Lage, die Patrozinien und Regeln (Funktionen) der von Karl IV. - gleichsam im Rhythmus seiner aufeinanderfolgenden Krönungen ${ }^{32}$ - gestifteten

31 Der Ausbau des hauptstädtischen Zentrums durch Gründung einer neuen Kommune (Stadtgemeinde) war im 14. Jahrhundert in Mitteleuropa keine neue Idee. Karl IV. konnte auf sie zurückgreifen und unter anderen dem Vorbild der zeitnahen Stiftung des polnischen Königs Kasimirs des Großen folgen, der im Jahr seines Herrschaftsantritts (1333) neben seiner Hauptstadt Krakau eine neue Stadt gründete und dieser seinen Namen verlieh. Zu diesen Stiftungen in Mitteleuropa im 13. und vor allem im 14. Jahrhundert siehe M. Słoń, Miasta podwójne i wielokrotne $w$ średniowiecznej Europie, (2011), S. 285-332.

321346 (in Bonn) und 1349 (in Aachen) wurde Karl zum römisch-deutschen König gekrönt, 1347 zum König von Böhmen, 1355 (in Mailand) zum König von Italien sowie (in Rom) zum Kaiser, und 1365 empfing er die Krone von Burgund (roi d'Arles). Der Zusammenhang der Gründung zumindest dreier Stiftungen (der beiden ersten sowie der Augustianer, oft auch der ambrosianischen Benediktiner) mit seinen Krönungen wurde in der älteren einschlägigen Literatur oft stark hervorgehoben. Zweifel an diesem Zusammenhang (außer was die Augustianer betraf) erhob Z. Hledíkova, Fundace českých králů ve 14. Století, „Sbornik Historický", 28 (1981), S. 5-55, S. 20, wobei sie auf die missionarische Funktion der slawischen Benediktiner und Karmeliter verwies. P. Crossley, The Politics of Presentation: The Architecture of Charles IV of Bohemia, in: Courts and regions in Medieval Europe, hg. v. S. Rees Jones, R. Marks, A. J. Minnis, (2000), S. 99-172, hier S. 129, machte jedoch zum Beispiel darauf aufmerksam, dass das Kloster mit slawischer Liturgie, das sich am Südeingang zur Stadt befand, eine Rolle in der königlichen Prozessionsliturgie spielte. Hundert Jahre nach dem Tod Karls IV. schrieb Sigmund Meisterlin, dass der Kaiser in Prag Klöster aller Orden haben wollte (Sigmund Meisterlins Chronik der Reichstadt Nürnberg, 1488, in: Die Chroniken 
sechs Klöster ${ }^{33}$. Auf dem höchsten Punkt - der hinsichtlich seiner Höhe gleichzeitig ein Äquivalent Wyschehrads war - errichtete er das Kloster und die Unserer Lieben Frau und Karl dem Großen gewidmete Augustianerkirche, eine besonders privilegierte Stiftung, die als symbolische Manifestierung der Verbindung Böhmens mit dem Reich gedacht war. Diese Kirche wurde auf dem Plan eines Oktogons und auf der Ost-West-Achse errichtet und knüpfte gleichzeitig an das Jerusalemer Vorbild wie an das der Aachener Kapelle $a^{34}$. Die vier Klöster bildeten ein Kreuz, in dessen Mitte, an der Schnittstelle seiner Arme, Karl das Kollegiatstift St. Apollinaris mit zehn Kanonikern stiftete, das eng mit der Universität verbunden war. Auf dem Plan der Neustadt lassen sich vier Teile unterscheiden:

1. der Mittelteil mit einer sich deutlich abzeichnenden Geometrie, dessen Achse der lange Platz bildete (der etwas später als Koňský trh [Rossmarkt]) bezeichnet wurde - der heutige Václavské náměstí [Wenzelsplatz];

2. der benachbarte Teil mit einem zentralen weiträumigen Platz (Dobytčí trh [Viehmarkt], heute Karlovo náměstí [Karlsplatz]); im Nordteil dieses Platzes stand das Neustädter Rathaus;

der fränkischen Städte. Nürnberg, 3, (Die Chroniken der deutschen Städte vom 14. bis ins 16. Jahrhundert 3, 11864, $\left.{ }^{2} 1961\right)$, S. 1-256, hier S. 156.

33 Diese Stiftungen und die Lage dieser Klöster besprach zuletzt Jan Pařez in zwei Artikeln: Kláštery na Novém Městě pražském do husitských válek a jejich právní a ekonomické postavení, in: Nove Město pražske, S. 75-91 sowie ders., Kláštery na Novém Městě pražském do husitských válek. Přispěvek k jejich historické geografii, in: Klasztor w mieście średniowiecznym i nowożytnym, hg. v. M. Derwich, A. Pobóg-Lenartowicz, (Opera ad historiam monasticam spectantia, series I: Colloquia 4, 2000), S. 235-241. Auf diesem Gelände befand sich, nachdem die Dominikaner und die Kreuzherren mit dem roten Stern noch im 13. Jahrhundert in die Altstadt umgezogen waren, nur ein Kloster - das des Ritterordens vom Heiligen Grab zu Jerusalem.

34 Karl IV. war fasziniert von der Idee der renovatio imperii. Karl der Große wird in seiner Autobiographie als praedecessor noster bezeichnet. Ähnliche Ideen verwirklichte er in seiner kaiserlichen Residenz in Ingelheim, in der er ein dem hl. Wenzel gewidmetes Oratorium stiftete, das eine Filiale des Prager Augustianerklosters bildete. 
3. der Nordteil mit seiner Hauptachse, der heutigen Hybernské nádraží;

4. der am stärksten hügelige südöstliche Teil, wo sich Weinberge und Gärten befanden.

Der Rossmarkt war durch ein Tor mit zwei Türmen verschlossen. Auch eine der aus dem zweiten großen Platz (dem Viehmarkt) hervorgehenden Achsen, die Gerstengasse [Ječná ulice], endete an einem massiven Stadttor (dem „Davids-Turm“). Diese Straße kreuzte die vom Wenzelsplatz nach Süden verlaufende Stephanstraße [Štěpánská ulice]. In der Mitte des späteren Viehmarktes errichtete Karl eine Fronleichnamskapelle. Seit 1351 wurden an diesem Ort in einem an die Holzkirche angebauten Turm die kaiserlichen Reliquien ausgestellt.

Das wäre also eine Aufteilung, die sich nicht nur auf die generelle Idee und den Symbolismus berief, wie er der vorherigen Epoche eigen war, dem dann also nur eine konkretere Form, ein physisches Element verliehen worden wäre, sondern eine Planung durch Einbringung von ostensiones der Passionsreliquien und zugleich der Reichskleinodien in die Topografie der Neustadt, d.h. die Manifestierung eines christologisch orientierten kaiserlichen Universalismus ${ }^{35}$. Wenn P. Crossley die Bedeutung der Ost-West-Achse bespricht, die mit der Gerstengasse begann und sich dann bis zum Tor des Heiligen Johannes hinzog, dann betont er die Dominanz der Fronleichnamskirche (Ort der Ausstellung der Reliquien, ein Prager Äquivalent des „Tempels Christi“) in der Mitte dieser Achse und den Sättigungsgrad ihrer memoriae: mit der neu errichteten Stephanskirche neben der alten Rotunde mit diesem Patrozinium, das in St. Longinus umgeändert wurde, und der Jerusalemkapelle (Grabkapelle, für verstorbene Pilger?) südöstlich der Kirche St. Stephan. Hier findet er Ähnlichkeiten zwischen der sakralen Topo-

35 Siehe H. Kühne, Ostensio reliquiarum. Untersuchungen über Entstehung, Ausbreitung, Gestalt und Funktion der Heiltumsweisungen im römisch-deutschen Regnum, (2000), S. 121, 122. Vgl. auch Kotrba, Nové Město pražské, S. 55-59; F. Kavka, K otázce sjednocení pražských měst v letech 1368-1377 a k místu Prahy v Karlově státní koncepci, „Documenta Pragensia“, 4 (1984), S. 100-120 (insbesondere S. 102). 
grafie und der schematischen (auf mittelalterlichen Plänen Jerusalems sichtbaren) Raumaufteilung des Jerusalems der Pilgerfahrten ${ }^{36}$.

Für die Einführung einer solchen Orientierung sprechen auch Vilém Lorenc zufolge in der Planung der Neustadt angewandten Jerusalemer Maße. Genauer gesagt, die Jerusalemer Distanzen, was schon von einer neuen, sich unter dem Einfluss der spätmittelalterlichen Wallfahrten herausbildenden Perzeption Jerusalems zeugt: die Jerusalemer Nord-Süd-Achse (vom Damaskustor bis zum Zionstor) beträgt 960 Meter, in Prag sind es auf derselben Achse vom Rossmarkt bis zum Augustinerkloster mit Kirche St. Katharina 948 Meter. Die Ost-WestAchse ist in Jerusalem 865 Meter lang, während es in Prag von seinem „Davids-Türmen“ in der Gerstengasse bis zum alten Weg nach Westen vom Viehmarkt, dem Weg „na Zderu“, 860 Meter sind. In Jerusalem beträgt der Abstand vom Zionstor zum Kettentor [Bab el-Silsileh], das zum Tempel hinführt, 582 Meter, und in Prag sind es zwischen dem Tor in der Gerstengasse und dem steinernen Tisch auf dem Karlsplatz ebenfalls $582 \mathrm{Meter}^{37}$. Allerdings kann nicht ausgeschlossen werden, dass diese Transplantation der Distanzen des historischen Jerusalems lediglich ein Produkt unserer Überinterpretation ist.

36 Crossley, The Politics of Presentation, S. 130 f. Allerdings fügt er hinzu: „Whether these correspondences of sacred geography where the results of conscious design or inspired coincidence, a more critical issue is the axial and progressive planning which the two cities share, conceptual in one, realized in the other. Writ large in Charles's New Town, in both its, sacred axes', an alliance in which the deposition of sacred sites and accents could be experienced, on a heroic scale, as historical progression and devotional fulfillment“ (S. 132). In der Prager Agglomeration kam es unter der Herrschaft Karls IV. zu noch einer weiteren, allerdings nicht topographischen Angleichung Prags an Jerusalem. Johannes Milicius von Kremsier [Jan Milíč z Kroměříž] gründete 1372 an der Stelle des „Schandhauses“ („Venice“ genannt) eine religiöse Gemeinschaft (u.a. für büßende Prostituierte) mit dem Namen Jerusalem, siehe D. Ch. Mengel, Bones, Stones and Brothels: Religion and Topography in Prague under emperor Charles IV (1346-78), (2003) [<http://etd.nd.edu/ETD-db/theses/available/etd-07282003-094532/unrestricted/etd.pdf >, besucht am 20.01.2017], S. 211-262. Matthias von Janov [Matěj z Janova] schrieb sogar, Milíč habe Prag aus einem Babylon in ein neues Jerusalem verwandelt.

37 Lorenc, Nové Město pražské, S. 52. 
Ungefähr um die Mitte der sechziger Jahre des 14. Jahrhunderts war in der Neustadt bereits der volle Umriss der sakralen Geografie der gesamten Anlage zu erkennen, der die sakrale Geografie der gesamten Agglomeration vom Hradschin bis nach Wyschehrad einerseits vervollständigte, aber auch umdefinierte, und andererseits eine Manifestierung der Position Prags im gesamten christlichen Universum darstellte. Karls Programm widerspiegelte wohl oder übel auch eine neue Perzeption Jerusalems nach den Kreuzzügen, in welcher der physische Kontakt, das materielle Konkretum dominierte, auch wenn dies eher eine historische Rekonstruktion der heiligen Stadt war als ihr zeitgenössisches Bild. Zur Angleichung Prags an Jerusalem genügte das dieses symbolisierende Kreuz der Kirchen nicht mehr; notwendig wurden die Jerusalemer Maße, die kopierten Hauptachsen der Stadt und sogar die topographischen Namen.

Görlitz ist wahrscheinlich das aus diesem Teil Europas bestbekannte Beispiel eines relativ frühen „Neuen Jerusalems“. An der Wende vom 15. zum 16. Jahrhundert wurde direkt vor der Stadt, unter Anwendung der Jerusalemer $\mathrm{Maße}^{38}$, auf einem abgeschlossenen und umzäunten Gelände ein Gebäudekomplex errichtet, bestehend aus der zweistöckigen Kreuzigungskapelle, der Grabeskapelle und der Salbungskapelle. Zusammen mit dem Kreuzweg und dem benachbarten symbolischen Ölberg bildete er Jerusalem nach. Ähnliche Kalvarienberge entstanden zur gleichen Zeit auch in einigen anderen deutschen Städten: 1488 in Berlin (auf dem dortigen Golgatha wurde eine hölzerne Grabeskapelle errichtet), 1490 in Nürnberg, 1500 in Bamberg und danach auch in Lübeck, Nördlingen, Halle, Trier usw. Von einigen dieser Unterneh-

38 Hans von Mergenthal, der im Jahre 1476 eine Pilgerfahrt ins Heilige Land unternahm, erwähnt in seinem Bericht zwei Gefährten seiner Wallfahrt aus Görlitz, s. Curiositäten der physisch-literarisch-artistisch-historischen Vor- und Mitwelt, hg. v. C. A. Vulpius, 3 (1814), S. 486-496, hier S. 488: „Diese zwei Eheleute haben das Muster vom heiligen Grabe zu Jerusalem genommen und darnach zu Görlitz vor der Stadt eine Kapelle lassen bauen und ein Grab in aller Gestalt wie das Heilige Grab zu Jerusalem ist". 
mungen wissen wir, dass ihnen bereits früher durchgeführte Kreuzwegandachten vorausgingen. Diese Repliken Jerusalems aus dem 15. Jahrhundert imitieren die wichtigsten Stationen des sanctus circuitus in Jerusalem: den Abendmahlssaal, Gethsemani, den Ölberg, den Gang durch den Bach Kidron, den Kreuzweg vom Prätorium (diese Rolle erfüllte in Görlitz, ähnlich wie anderswo auch, die Pfarrkirche St. Peter) und Golgatha. Wir wissen nicht genau, welchen Weg die Prozession damals in Görlitz zurückgelegt hat. Wir kennen ihn nur aus späteren Zeiten. Seine wichtigste Etappe bildete der ,in Übereinstimmung“ mit der Jerusalemer Distanz abgemessene Kreuzweg mit einer Länge von 970 Schritten $^{39}$.

Noch anders wurde die Nachbildung Jerusalems in Breslau verwirklicht. Die Grundlage dafür bildete im 15. Jahrhundert die Liturgie des Palmsonntags und der Karwoche. Die größten Menschenmassen zog die Prozession am Palmsonntag an, die am Morgen von der Kathedrale (außerhalb der Stadtmauer auf der Oderinsel) zur Kirche Unserer Lieben Frau (welche auch schon außerhalb der Stadt auf der sogenannten Sandinsel lag) und wieder zurück führte. Sie bestand aus zwei Teilen: der erste imitierte den Einzug Jesu in Jerusalem, während der zweite - auf dem Rückweg - an den Kreuzweg anknüpfte. Aus einer Beschreibung der Prozession im Jahre 1423 (sie entstand, weil damals unter dem Andrang der Menge die Brücke eingestürzt war) geht hervor, dass sie unter Verwendung eines in deutschen Städten populären sogenannten Palmesels stattfand. Auf dem Rückweg, schon nach der Weihe der Palmen und ihrer Verteilung unter die Gläubigen, machte die Prozession auf dem Platz vor der Stiftskirche zum Heiligen Kreuz Halt. An diesem Ort wurde die Station sanctae crucis zelebriert. Wichtig ist hierbei die Topografie der außerhalb der Stadtmauern gelegenen Inseln in der Oder sowie die Patrozinien der Kirchen: die dem hl. Jo-

39 Siehe G. Roth, Das „Heilige Grab“ in Görlitz, in: Der Jakobuskult in Ostmitteleuropa. Austausch - Einflüsse - Wirkungen, hg. v. K. Herbers, D. R. Bauer, (Jakobus-Studien 12, 2003), S. 259-284. 
hannes dem Täufer und dem hl. Evangelisten Johannes geweihte Kathedrale auf der Dominsel, am anderen Ende dieser Insel die zweistöckige Stiftskirche zum Heiligen Kreuz, und dann die Augustianerkirche zur Allerheiligsten Jungfrau Maria auf der Sandinsel. Auf der Ost-WestAchse gelegen, bilden sie aus ihren Patrozinien ein Triumphkreuz ab. In derselben Aufstellung - von Seiten der Stadt aus gesehen - in der in der Kirche die Szene unter dem Kreuz von Seiten des Langhauses aus betrachtet wurde.

Die Prager Neustadt war nicht nur die älteste dieser drei Unternehmungen, sondern sie resultierte auch aus urbanistischen Konzeptionen, deren Beginn in der Zeit der Wende von der Antike zum Mittelalter lag. Dabei handelt es sich um die Realisierung einer symbolischen Topografie, die auch schon spätmittelalterliche Elemente enthält, wie dies vielleicht in der Nachbildung realer Jerusalemer Distanzen zum Ausdruck kommt. Der anamnetische Charakter solcher Realisierungen resultierte aus der Perzeption des Vorbilds - in diesem Falle Jerusalems - sowie aus dem Konzept der Replik (der Kopie), welche auf der Nachbildung bedeutsamer Elemente beruhte, die sowohl das Original als auch die Imitation (ad instar) konstituierten: die Patrozinien der Kirchen, ihre Lage, insbesondere innerhalb oder außerhalb (besonders der Stadtmauern) sowie zueinander, die ein Zeichen des Kreuzes schufen usw. Dies war somit eine Replik der sakralen Topografie, die im Spätmittelalter auch um konkrete Dinge angereichert wurden, wie die Jerusalemer Maße oder Distanzen. In gewissem Sinne stellte die Prager Neustadt auch eine Reaktion auf die römische Replik dar, wie sie das Residenz- und Kirchenensemble auf dem Wyschehrad darstellte, aber sie bildete auch deren Ergänzung, so dass auf diese Weise die gesamte Prager Agglomeration zu einer außergewöhnlichen urbanistischen Anlage wurde. Das Breslauer „Jerusalem“ hatte anderen Charakter, andere Struktur und Funktion. Es entstand aus dem spätmittelalterlichen Passionskult und bestimmten Formen seiner Zelebrierung (Passionsspiel, Kreuzwegandacht), die nicht so sehr die anamnetische Kraft der Jerusalemer Topografie offenbarten als vielmehr die der Geschehnisse, 
die sich in ihr abspielten. Die realistische, materielle Nachahmung dieser Geschehnisse in den alljährlichen Feierlichkeiten der Karwoche schufen eine Illusion der Teilnahme an ihnen, der Versetzung in Raum und Zeit zu den historischen Orten und Fakten, welche jedoch mit einer enormen Aufladung apokrypher Konkreta ohne Distanz zur Vergangenheit und der sich daraus ergebenden plastischen Darstellungen und Vorstellungen umgeben wurden. Derartige „Repliken“ Jerusalems hatten ephemeren (wenn auch wiederholbaren) Charakter, der städtische oder der kirchliche Raum spielte hier nur die Rolle einer Bühne und musste daher nicht an die Topografie Jerusalems anknüpfen oder die Grabeskirche nachbilden. Und das Görlitzer Jerusalem gehört wenn wir von der außergewöhnlichen Gründung von Santo Stefano in Bologna und anderen innerhalb der städtischen Agglomeration gelegenen Klostergründungen absehen - zu den relativ frühen städtischen Anknüpfungen an die Jerusalemer Topografie, die unter dem Einfluss von Pilgerfahrten ins Heilige Land entstanden. Sie enthielten sowohl die ältesten Elemente der Repliken der Grabeskirche (die Architektur der Grabeskapelle, die Zweistöckigkeit der Kreuzigungskapelle, die Maße usw.) als auch ihre neuen, mit dem Kult der Passion Christi verbundenen Funktionen. Diese „treue“ Kopie (um hier an die Worte von Hans Mergenthal anzuknüpfen) der wichtigsten Heiligtümer in der Grabeskirche und ihre Aufstellung in deren Inneren enthielt eine materielle Wortwörtlichkeit, wie sie dem Breslauer Jerusalem fehlte.

Ein Vergleich dieser drei an Jerusalem anknüpfenden Gründungen führt zu dem Schluss, dass sich im Spätmittelalter eine wichtige Veränderung vollzog: Die sakrale Topografie machte einer sakralen Geografie Platz, die ausschließlich durch den concursus populi und den Prozessionsritus aktuell, lebendig und real ist. Im Breslauer Jerusalem wurde er noch ohne buchstäbliche Wiederholung des Jerusalemer circuitus wiedergegeben, während nach Görlitz die Prozession verlegt wurde, in der die Pilger die Grabeskirche betraten, und später auch das ganze Jerusalemer Anwesen. Ich brauche nicht hinzuzufügen, dass unter dem Einfluss der Veränderungen, die im Passionszeremoniell in Europa 
erfolgten, auch diese Jerusalemer Umgebung evolvierte. Jede Replik ist ein Kommentar und zeugt sowohl von der Perzeption des Originals als auch vom Verständnis der Beziehungen zwischen ihm und seiner Replik. Ein Vergleich dieser drei Verwirklichungen erlaubt die Wende zu verstehen, die im 15. Jahrhundert in diesem Teil Europas stattfand, an einem Ort, den der Passionskult im religiösen und sozialen Leben einnahm. Er gibt einerseits gut den immer marginaleren Stellenwert wieder, den die symbolische Perzeption in der spätmittelalterlichen Stadtkultur einnahm, andererseits aber auch das Bedürfnis nach einem materiellen Konkretum, nach Betrachten und Berühren, um zu verstehen und in Erinnerung zu behalten.

Das bedeutet zum Schluss nichts anderes, als dass bei der Darstellung der sakralen Topografie der Stadt ein flacher Plan nicht ausreicht, man braucht mehr Dimensionen, Vielfältigkeit und Dynamik. Dies betrifft in gleicher Weise den Text, also Beschreibung und Begründung des rekonstruierten Raumes, und die Karte. Beide Darstellungsweisen müssen eng verknüpft werden.

Korrekturgelesen von Dirk Rosenstock

\section{PONAD DWA WYMIARY}

GEOGRAFIA SAKRALNA MIASTA ŚREDNIOWIECZNEGO „DO WEWNĄTRZ” ORAZ „WZWYŻ"

\section{(STRESZCZENIE)}

Autorzy odróżniają pojęcie „geografia sakralna” od określeń „topografia sakralna” i „topografia kościelna” (w znaczeniu ,instytucjonalno-społecznym”). W artykule podkreślono więc znacznie percepcji przestrzeni i czasu, przeprowadzono analizę relacji między tymi kategoriami oraz rozważano zjawiska symbolicznej manifestacji sacrum (hierofania). Geografia sakralna jest tu rozumiana jako kategoria społecznych wyobrażeń, praktyki i percepcji, która w stopniu co najmniej znaczącym wpływa na kształt miasta. Autorzy odwołują się do przykładów najsłynniejszych (najświętszych) miast: Jerozolimy, Rzymu i Akwizgranu i ich oddziaływania na miasta Europy Środkowo-Wchodniej. 


\section{ÜBER ZWEI DIMENSIONEN HINAUS}

\section{DiE SAKRALE GEOGRAFIE DER MITTELALTERLICHEN \\ STADT „NACH INNEN“ UND „NACH OBEN"}

\section{(ZUSAMMENFASSUNG)}

Die Autoren unterscheiden den Begriff „sakrale Geografie“ von den Bezeichnungen „sakrale Topografie“ und „kirchliche Topografie“ (als institutionell-soziale). Im Artikel wurde dann die Bedeutung der Perzeption von Raum und Zeit betont, eine Analyse der Zusammenhänge zwischen diesen Kategorien durchgeführt und das symbolische Aufscheinen des Heiligen im Profanen (Hierophanie) überlegt. Die sakrale Geografie wurde damit als eine Kategorie sozialer Vorstellungen, Praxis und Perzeption genannt, die in zumindest wesentlichem Maße Einfluss auf die Gestalt der Stadt hat. Die Autoren berufen sich auf die Beispiele der berühmtesten (heiligsten) Städte: Jerusalem, Rom und Aachen und ihren Einfluss auf die Städte Ostmitteleuropas.

\section{MORE THAN TWO DIMENSIONS}

\section{THE SACRED GeOgRaphy OF THE MEDIEVAL TOWN "INSIDE" AND "UPWARDS"}

(SUMMARY)

The authors distinguish the term "sacred geography" from the terms "sacred topography" and "church topography" (in the institutional-social sense). The article underlines the importance of the perception of space and time; it includes the analysis of the relations between those categories and analyzes the phenomena of social images, practices and perceptions, which affect the town. The authors refer to the examples of the most famous (the most holy) towns of Jerusalem, Rome and Aachen and their influence on the towns in Central and Eastern Europe.

Translated by

Agnieszka Chabros

\section{Słowa kluczowe / Schlagworte / Keywords}

- średniowiecze; geografia sakralna; przestrzeń miejska; świętość

- Mittelalter; sakrale Geografie; urbaner Raum; Heiligtum

- the Middle Ages; sacred geography; urban space; holiness 


\section{BIBLIOGRAFIA / BIBLIOGRAFIE / BIBLIOGRAPHY}

\section{ŹRÓDłA DRUKOWANE / GEDRUCKTE QUELLEN / PRINTED SOURCES}

Adalberti Vita Heinrici II imperatoris, hg. v. G. Waitz, in: Annales, chronica et historiae aevi Carolini et Saxonici, hg. v. G. H. Pertz, (Monumenta Germaniae Historica, Scriptores 4, 1841), cap. 7, S. 794.

Canonici Wissegradensis continuatio Cosmae, hg. v. J. Elmer, (Fontes rerum Bohemicarum 2, 1874), S. 201-237.

Curiositäten der physisch-literarisch-artistisch-historischen Vor- und Mitwelt, hg. v. C. A. Vulpius, 3 (1814).

Das Constitutum Constantini, hg. v. H. Fuhrmann, (Monumenta Germaniae Historica, Fontes iuris Germanici antiqui, in usum scholarum 10, 1968), S. 56-98. Iohannis Beleth, Summa de ecclesiasticis officiis, hg. v. H. Douteil, (Corpus Christianorum Continuatio Maedievalis $41 \mathrm{~A}, 1975)$.

Isidori Hispalensis episcopi Etymologiarum sive Originvm libri XX, hg. v. W. M. Lindsay, 1 (1911).

Isidoro de Sevilla. Diferencias, libro I. Introducción, edición crítica, traducción y notas, hg. v. C. Codoñer, (Auteurs Latins du Moyen Âge 1992).

Sigmund Meisterlins Chronik der Reichstadt Nürnberg, 1488, in: Die Chroniken der fränkischen Städte. Nürnberg, 3 (Die Chroniken der deutschen Städte vom 14. bis ins 16. Jahrhundert $\left.3,{ }^{1} 1864,{ }^{2} 1961\right)$, S. $1-256$.

\section{LITERATURA / LITERATUR / LITERATURE}

Arnold K., Bilder und Texte. Stadtbeschreibung und Städtelob bei Hartmann Schedel, in: Acta Conventus Neo-Latini Hafniensis. Proceedings of the Eighth International Congress of Neo-Latin Studies, (Medieval Texts and Studies 120, 1994), S. 121-132.

Bek L., The Changing Architectonic Aspect of the Ideal City in the Early Renaissance, in: Acta Conventus Neo-Latini Hafniensis. Proceedings of the Eighth International Congress of Neo-Latin Studies, (Medieval Texts and Studies 120, 1994), S. 143-153.

Brown P., The Cult of the Saints: Its Rise and Function in Latin Christianity,(1981).

Certeau M. de, L'Invention du quotidien, 1: Arts de faire (1990).

Crossley P., The Politics of Presentation: The Architecture of Charles IV of Bohemia, in: Courts and regions in Medieval Europe, hg. v. S. Rees Jones, R. Marks, A. J. Minnis, (2000), S. 99-172. 
Delehaye H., Loca sanctorum, (1930).

Friedman D., 'Fiorenza' Geography and Representation in a Fifteenth Century City View, „Zeitschrift für Kunstgeschichte“, 64 (2001), S. 56-77.

Halbwachs M., La topographie légendaire des Évangiles en Terre sainte. Étude de mémoire collective, (1941).

Hledíkova Z., Fundace českých králi̊ ve 14. Století,„Sbornik Historický“, 28 (1981), S. 5-55.

Hochmuth C., Rau S., Stadt - Macht - Räume. Eine Einführung, in: Machträume der frühneuzeitlichen Stadt, hg. v. C. Hochmuth, S. Rau, (2006), S. 13-40.

Iogna-Prat D., La Maison Dieu. Une histoire monumentale de l'Église au Moyen Âge (v. 800-v. 1200), (2006).

Kavka F., K otázce sjednocení pražských měst v letech 1368-1377 a k místu Prahy $v$ Karlově státní koncepci, „Documenta Pragensia“, 4 (1984), S. 100-120.

Kotrba V., Nové Město pražské: „Karlstadt“ v univerzální koncepci císaře Karla $I V$., in: $Z$ tradic slovanské kultury $v$ Čechách (Sázava a Emauzyv dějinách české kultury), hg. v. J. Petr, S. Šabouk, (1975), S. 53-66.

Kühne H., Ostensio reliquiarum. Untersuchungen über Entstehung, Ausbreitung, Gestalt und Funktion der Heiltumsweisungen im römisch-deutschen Regnum, (2000).

Lauwers M., La mémoire des ancêtres. Le souci des morts. Morts, rites et société au Moyen Âge, (1966).

Ledvinka V., Pešek J., Prag, (2000).

Lefebvre H., La production de l'espace, (1974).

Lehmann-Brockhaus O., Schriftquellen zur Kunstgeschichte des 11. und 12. Jahrhunderts für Deutschland, Lotharingen und Italien, (1938).

Lorenc V., Das Prag Karls IV: Die Prager Neustadt, (1982).

Lorenc V., Nové Město pražské, (1973).

Luoghi sacri e spazi della santità, hg. v. S. Boesch Gajano, L. Scaraffia, (1990).

Manikowska H., Sacred Geography of a Town, „Acta Poloniae Historica“, 101 (2010), S. 51-87.

Méhu D., Locus, transitus, peregrinatio. Remarques sur la spatialité des rapports sociaux dans l'Occident médiéval (Xie-XIIIe siècle), in: Construction de lespace au Moyen Âge: pratiques et représentations. XXXVIIe Congrès de la SHMES (Mulhouse, 2-4 juin 2006), (2007), S. 275-293.

Mengel D. Ch., Bones, Stones and Brothels: Religion and Topography in Prague under emperor Charles IV (1346-78), (2003).

Michałowski R., Princeps fundator. Studium $z$ dziejów kultury politycznej w Polsce X-XIII wieku, (1993). 
Morelowski M., Początki świadomej myśli urbanistycznej w Polsce przed kolonizacja XIII w., in: Sztuka i historia. Ksiega pamiątkowa ku czci profesora Michała Walickiego, (1966), S. 39.

Morlet V., Recueil de textes relatifs à l'histoire de l'architecture et à la condition des architectes en France au moyen âge, XI-XII siècles, (1911).

Nové Město pražské ve 14.-20. století, hg. v. V. Ledvinka, J. Pešek, „Documenta Pragensia“, 17 (1998).

Pařez J., Kláštery na Novém Městě pražském do husitských válek a jejich právní a ekonomické postavení, in: Nové Město pražské ve 14.-20. století, hg. v. V. Ledvinka, J. Pešek, „Documenta Pragensia“, 17 (1998), S. 75-91.

Pařez J., Kláštery na Novém Městě pražském do husitských válek. Přispěvek $k$ jejich historické geografii, in: Klasztor $w$ mieście średniowiecznym i nowożytnym, hg. v. M. Derwich, A. Pobóg-Lenartowicz, (Opera ad historiam monasticam spectantia, series I: Colloquia 4, 2000), S. 235-241.

Pauly M., Scheutz M., Der Raum und die Geschichte am Beispiel der Stadtgeschichtsforschung, in: Cities and their spaces: Concepts and their use in Europe, hg. v. M. Pauly, M. Scheutz, (Stadtforschung A 88, 2014), S. 1-14.

Pleszczyński A., Przestrzeń i polityka. Studium rezydencji władcy wcześniejszego średniowiecza. Przykład czeskiego Wyszehradu, (2000).

Roth G., Das „Heilige Grab“ in Görlitz, in: Der Jakobuskult in Ostmitteleuropa. Austausch - Einflüsse - Wirkungen, hg. v. K. Herbers, D. R. Bauer, (JakobusStudien 12, 2003), S. 259-284.

Schlosser J. von, Quellenbuch zur Kunstgeschichte des abendländischen Mittelalters, (1896).

Skwierczyński K., Custodia civitatis. Sakralny system ochrony miasta w Polsce wczesnego średniowiecza na przykładzie siedzib biskupich, „Kwartalnik Historyczny", 53, 3 (1996), S. 3-51.

Słoń M., Miasta podwójne i wielokrotne w średniowiecznej Europie, (2011), S. 285-332.

Souza M. de, La question de la tripartition des catégories $d u$ droit divin dans l'Antiquité romaine, (2004).

Vaníček V., Vratislav II. (I.) - První český král. Čechy v době evropského kulturního obratu $v$ 11. Století, (2004).

Witkowska A., Kulty pątnicze piętnastowiecznego Krakowa. Z badań nad miejską kulturą religijną, (1984). 